
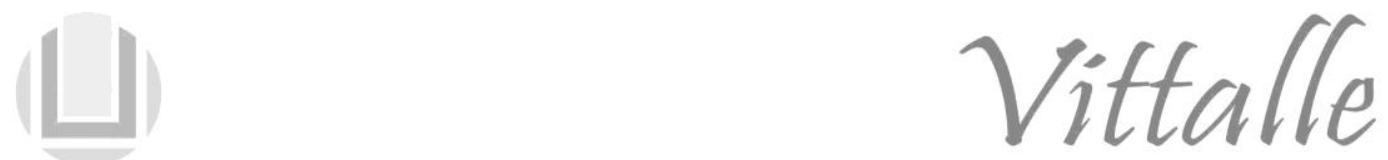

\title{
Transtornos mentais comuns e aspectos psicossociais em universitários do sul do Brasil
}

\author{
João Paulino Perini ${ }^{\mathrm{a},{ }^{*}}$, Eslen Delanogare ${ }^{\mathrm{a},{ }^{*}}$, Sabrina Alves de Souza ${ }^{\mathrm{b}}$ \\ ${ }^{\text {a} P r o g r a m a ~ d e ~ P o ́ s-G r a d u a c ̧ a ̃ o ~ e m ~ N e u r o c i e ̂ n c i a s, ~ N e u r o s c i e n c e ~ C o w o r k i n g ~ L a b, ~ U n i v e r s i d a d e ~ F e d e r a l ~ d e ~ S a n t a ~}$ \\ Catarina, Florianópolis, SC, Brasil \\ ${ }^{\mathrm{b}}$ Departamento de Ciências Humanas, Universidade Regional Integrada do Alto Uruguai e das Missões, Santo Ângelo, \\ RS, Brasil
}

Histórico do Artigo

Recebido em:

$19 / 01 / 2019$

Aceito em:

$23 / 03 / 2019$

Palavras-chave:

Transtorno Mental

Comum;

Universitários; Saúde

Mental.

\begin{abstract}
RESUMO
Os transtornos mentais caracterizam-se como um dos principais fatores responsáveis pelo aumento da morbimortalidade em nível global, principalmente devido à natureza crônica e incapacitante da depressão e outros transtornos mentais comuns (TMC). No entanto, o tratamento dos TMC é um desafio. Nesse sentido, o presente trabalho pretende contribuir com dados acerca da prevalência dos TMC e associação com aspectos psicossociais em estudantes de uma universidade de Santo Ângelo - RS. A amostra foi composta por um total de 246 alunos. A prevalência de TMC na amostra foi de $40 \%$, resultado semelhante a outros estudos. Os fatores associados à presença de TMC foram: gênero, insatisfação com o curso, dificuldade em fazer amigos ou sentir-se rejeitado por eles, percepção de pouco apoio emocional. Outro fator relevante foi o não recebimento de atendimento em saúde mental, que compõe $88,8 \%$ dos sujeitos com TMC. Esses resultados mostram que podem ser desenvolvidas estratégias de prevenção e tratamento de saúde mental em ambiente acadêmico. O presente trabalho demonstrou a presença de TMC em uma população universitária e variáveis associadas.
\end{abstract}

Common mental disorders and psychosocial aspects in university students from southern brazil

\section{ABSTRACT}

Mental disorders are characterized as one of the main factors responsible for the increase in morbidity and mortality at the global level, mainly due to the chronic and incapacitating nature of depression and other common mental disorders (CMD). However, treating CMD is a challenge. In this sense, the present work intends to contribute with data about the prevalence of CMD and association with psychosocial aspects in students of a university of Santo Ângelo RS. The sample consisted of a total of 246 students. The prevalence of CMD in the sample was $40 \%$, similar to other studies. The factors associated with the presence of CMD were: gender, dissatisfaction with the course, difficulty in making friends or feeling rejected by them, perception of little emotional support. Another relevant factor was the non-receipt of mental health care, which makes up $88.8 \%$ of subjects with CMD. These results show that mental health prevention and treatment strategies can be developed in an academic setting. The present study demonstrated the presence of CMD in a university population and associated variables.

\section{Introdução}

Os transtornos mentais correspondem a aproximadamente 32,4\% dos Anos Vividos com Incapacidades e 13\% da Esperança de Vida Corrigida pela Incapacidade (1). O número total de pessoas convivendo com depressão, que corresponde a um dos transtornos mentais mais comuns, aumentou 18,4\% de 2005 a 2015 (2) e estima-se que 322 milhões de pessoas conviviam com a depressão em 2017 (3). Um importante avanço no campo da epidemiologia das doenças mentais foi dado pelo Global Burden

\footnotetext{
*Autores correspondentes: joaopaulinoperini@gmail.com (Perini J.P.); eslenneuro@ gmail.com (Delanogare E.)
} 
Disease através da aplicação de abordagens quantitativas que visam integrar descobertas de estudos epidemiológicos relacionados a doenças mentais, resultando em uma série de revisões sistemáticas e meta-análises sobre as prevalências dessas patologias (4). De fato, os transtornos mentais caracterizam-se como um dos principais fatores responsáveis pelo aumento da morbimortalidade em nível global, principalmente devido à natureza crônica e incapacitante da depressão e outros transtornos mentais comuns (TMC), transtornos relacionados ao uso de álcool e substâncias psicoativas e psicoses (5). Especificamente, os TMC possuem alta prevalência e afetam pessoas em todas as regiões do mundo (4). Os TMC configuram-se por transtornos depressivos e ansiosos, gerando impacto no humor ou sentimentos das pessoas afetadas, onde os sintomas variam em termos de gravidade de leve à grave, podendo durar de meses a anos (3). Por outro lado, o tratamento dos TMC é um desafio e tendo em vista o insucesso no desenvolvimento de novos fármacos que atuem em doenças psiquiátricas nas últimas décadas (6). Nas últimas décadas, têm-se optado pela união do uso de fármacos psiquiátricos, i.e fluoxetina, e Terapia Cognitiva Comportamental visando um tratamento mais efetivo $(7,8)$. Contudo, a heterogeneidade, o curso extremamente variável e o desconhecimento da etiologia desses transtornos dificultam a obtenção de êxito no processo terapêutico (9).

Nesse sentido, o presente trabalho pretende contribuir com dados acerca da prevalência dos TMC e a associação com aspectos psicossociais em estudantes de uma universidade privada de Santo Ângelo - RS.

\section{Material e método}

A presente pesquisa caracteriza-se como transversal, com abordagem quantitativa descritiva.

\subsection{Etapas do processo para obtenção da amostra:}

I- Levantamento, junto à universidade, do número total de alunos regularmente matriculados nos semestres ímpares (1.536 alunos) para o cálculo da amostra e posterior estratificação de acordo com os cursos de graduação;

II- Adoção do Intervalo de Confiança (IC) de 95\% com erro tolerável amostral de $6 \%$ e prevalência estimada de $50 \%$.

III- A fim de garantir a representatividade estatística, optou-se pelo acréscimo de $8 \%$ na amostra total em turmas com poucos alunos matriculados.

\subsection{Critérios de inclusão e estratificação}

Possuir 18 anos ou mais e estar cursando o $1^{\circ}, 5^{\circ}$ ou $9^{\circ}$ semestre, constituindo três grupos: iniciantes, intermediários e concluintes, respectivamente. Extraordinariamente para os cursos com finalização em quatro anos, a categoria de concluintes foi preenchida pelos alunos do $7^{\circ}$ semestre.

Objetivando testar o manejo da aplicação e a aceitabilidade do autopreenchimento, realizou-se um estudo piloto com $3 \%$ da amostra. Os sujeitos do estudo piloto não participaram da pesquisa e os dados não são apresentados no presente estudo.

\subsection{Instrumentos utilizados}

Para o rastreamento de TMC, foi utilizado o instrumento Self-Report Questionnaire (SRQ-20), desenvolvido por Harding (10) e validado no Brasil por Mari e Williams (11). O SRQ-20 é um instrumento utilizado para rastrear casos de TMC não psicóticos; contém 20 perguntas com respostas "sim" e "não", sendo que cada resposta afirmativa soma 1 ponto em uma escala de 0 a 20 . O ponto de corte que sugere a presença de TMC 
é $\geq 7$ respostas "Sim". Por ser de rápida aplicação, não requer entrevistador clínico e com alto poder de discriminação de casos, o SRQ-20 é recomendado pela OMS para pesquisas em saúde mental, principalmente em países em desenvolvimento (12).

Para a obtenção de variáveis sociodemográficas e de assistência em saúde mental, os autores desenvolveram um questionário próprio que incluiu questões sobre satisfação com a escolha profissional, dificuldade de fazer amigos, percepção de apoio emocional, percepção de rejeição por amigos, atendimento em saúde mental e variáveis sociodemográficas.

\subsection{Procedimento de coleta de dados}

Após a aprovação do projeto pelo comitê de ética, os pesquisadores contataram os coordenadores e professores dos respectivos cursos e agendaram os dias para aplicação dos instrumentos. Os estudantes que aceitaram participar da pesquisa leram o Termo de Consentimento Livre e Esclarecido (TCLE) e o assinaram em duas vias, uma ficando em sua posse e a outra depositaram em uma urna. A aplicação dos questionários foi realizada de forma coletiva nas salas de aula, com tempo médio de 10 minutos. Após responderem os instrumentos, os acadêmicos os depositaram em uma segunda urna de forma a garantir o sigilo.

\subsection{Considerações éticas}

Essa pesquisa foi desenvolvida com base em parâmetros éticos, atendendo à resolução $n^{\circ}$ 466, de 12 de dezembro de 2012 do Conselho Nacional de Saúde. Este estudo foi aprovado pelo Comitê de Ética e Pesquisa sob parecer 1.973.701. O TCLE foi entregue aos participantes e ressaltou-se o sigilo, a voluntariedade, a possibilidade de desistência a qualquer momento e de encaminhamento para atendimento psicológico caso o questionário resultasse em algum desconforto.

\subsection{Tratamento e Análise dos dados}

Para análise dos dados foi utilizado o programa Statistical Package for the Social Sciences (SPSS) versão 24. Primeiramente, foi realizada análise de consistência com o intuito de avaliar casos omissos, identificação de extremos e possíveis erros de digitação. Posteriormente, a análise descritiva foi usada para determinar o grupo com presença de TMC, considerando escore de $\geq 7$ respostas positivas "Sim" no instrumento SRQ-20. O teste Qui-quadrado foi usado para avaliar a associação entre as variáveis. O nível de significância adotado foi de $\mathrm{p}$-valor $\leq 0.05$.

\section{Resultados}

\subsection{Amostra}

A tabela 1 apresenta distribuição da amostra por área do conhecimento. A amostra foi composta por um total de 246 alunos distribuídos em 20 cursos de graduação.

Tabela 1- Distribuição da amostra por área de conhecimento, segundo diretrizes do CNPq.

\begin{tabular}{lcc|c}
\hline & Área & Total & \% \\
\hline Ciências Exatas e da Terra & 18 & 7,3 \\
Ciências Biológicas & & 10 & 4,1 \\
Engenharias & 57 & 23,0 \\
Ciências da Saúde & 43 & 17,0 \\
Ciências Agrárias & 6 & 2,4 \\
Ciências Sociais Aplicadas & 80 & 33,0 \\
Ciências Humanas & 32 & 13,0 \\
\hline
\end{tabular}


A amostra foi composta por $60,1 \%$ sujeitos do gênero feminino. A média de idade dos participantes foi de 21,5 anos ( $\pm 4,35) ; 35,8 \%$ eram iniciantes no curso de graduação; $63,4 \%$ residiam na cidade de estudo e metade dos estudantes moravam com os pais (Tabela 2).

Tabela 2- Perfil da amostra dos estudantes de uma universidade de Santo Ângelo - RS, 2017 $(\mathrm{N}=246)$.

\begin{tabular}{|c|c|c|}
\hline Variáveis & $\mathbf{N}^{\mathbf{o}}$ & $\%$ \\
\hline Total & 246 & 100 \\
\hline \multicolumn{3}{|l|}{ Gênero } \\
\hline Feminino & 148 & 60,1 \\
\hline Masculino & 98 & 39,9 \\
\hline \multicolumn{3}{|l|}{ Período } \\
\hline Iniciantes & 88 & 35,8 \\
\hline Intermediários & 83 & 33,7 \\
\hline Concluintes & 75 & 30,5 \\
\hline \multicolumn{3}{|l|}{ Reside na cidade que estuda } \\
\hline Sim & 156 & 63,4 \\
\hline Não & 90 & 36,6 \\
\hline \multicolumn{3}{|l|}{ Condições de moradia } \\
\hline Mora com os pais & 123 & 50,0 \\
\hline Paga aluguel sozinho (a) & 37 & 15,0 \\
\hline Divide aluguel & 35 & 14,2 \\
\hline Casa cedida & 11 & 4,5 \\
\hline \multicolumn{3}{|l|}{ Exerce atividade remunerada } \\
\hline Não & 122 & 49,6 \\
\hline Sim & 119 & 48,4 \\
\hline Não sabe & 5 & 2,0 \\
\hline
\end{tabular}

\subsection{Descrição dos dados e TMC}

A prevalência de TMC na amostra foi de 40,0\%, representando o total de 99 sujeitos. Na tabela 3 estão representadas a descrição dos dados em relação ao TMC. Os achados indicam maior prevalência em mulheres $(48,6 \%)$; em estudantes insatisfeitos com a escolha profissional (70,0\%); que pensam em abandonar o curso $(66,7 \%)$; que possuem dificuldade em fazer amigos (70,3\%); que têm sentimento de rejeição por amigos e colegas $(83,3 \%)$ e os que não recebem apoio emocional $(63,6 \%)$.

Tabela 3-Descrição dos aspectos psicossociais, gênero e Transtornos Mentais Comuns em estudantes de uma universidade de Santo Ângelo - RS, 2017 (N=246).

\begin{tabular}{lcccc}
\hline \multicolumn{1}{c}{ Variáveis } & $\mathbf{N}^{\mathbf{0}}$ & $\boldsymbol{\%}$ & $\mathbf{T M C}$ & $\boldsymbol{p}^{*}$ \\
\hline Gênero & & & & \\
$\quad$ Feminino & & & & 0,001 \\
$\quad$ Masculino & 148 & 60,1 & $48,6 \%$ & \\
Satisfação com a escolha profissional & 98 & 39.9 & $27,6 \%$ & \\
$\quad$ Sim & & & & 0,034 \\
$\quad$ Não & 223 & 90.6 & $37,7 \%$ & \\
$\quad$ Não sabe & 10 & 4,0 & $70,0 \%$ & \\
\hline
\end{tabular}


Vittalle - Revista de Ciências da Saúde v. 31, n. 1 (2019) 44-51

Continuação

\begin{tabular}{|lcccc}
\hline \multicolumn{1}{c}{ Variáveis } & $\mathbf{N}^{\mathbf{0}}$ & $\boldsymbol{\%}$ & $\mathbf{T M C}$ & $\boldsymbol{p}^{*}$ \\
\hline Já pensou em abandonar o curso & & & & 0,001 \\
$\quad$ Sim, ainda penso & 18 & 7,3 & $66,7 \%$ & \\
$\quad$ Sim, mas não penso mais & 84 & 34,1 & $51,2 \%$ & \\
$\quad$ Não, nunca & 144 & 58,5 & $30,6 \%$ & \\
Dificuldade em fazer amigos & & & & $<0,001$ \\
$\quad$ Sim & 37 & 15,0 & $70,3 \%$ & \\
$\quad$ Não & 203 & 82.5 & $35,0 \%$ & \\
$\quad$ Não sabe & 6 & 2,5 & $33,3 \%$ & \\
Sente-se rejeitado por amigos e colegas & & & & $<0,001$ \\
$\quad$ Sim & 18 & 7,4 & $83,3 \%$ & \\
$\quad$ Não & 219 & 89,0 & $36,5 \%$ & \\
$\quad$ Não sabe & 9 & 3,6 & $44,4 \%$ & \\
Sente que recebe apoio emocional & & & & $<0,001$ \\
$\quad$ Sim & 175 & 71,1 & $32,6 \%$ & \\
$\quad$ Não & 55 & 22,4 & $63,6 \%$ & \\
$\quad$ Não sabe & 16 & 6,5 & $43,8 \%$ & \\
& & & & \\
\hline
\end{tabular}

*Teste do Qui- quadrado

\subsection{Atendimento em saúde mental no Grupo com TMC}

$\mathrm{O}$ recebimento de atendimento profissional no grupo TMC é significativamente menor, isto é, $88,8 \%$ dos sujeitos do grupo TMC não recebem atendimento especializado em saúde mental.

Tabela 4 - Atendimento em saúde mental no Grupo TMC (n=99) em estudantes de uma universidade de Santo Ângelo - RS, 2017.

\begin{tabular}{lccc}
\hline \multicolumn{1}{c}{ Variável } & Total & \% TMC & $\boldsymbol{p}^{*}$ \\
\hline Recebe atendimento em saúde mental & & & 0,002 \\
Não & 80 & 88,8 & \\
Sim & 19 & 19,2 \\
\end{tabular}

* Teste do Qui-quadrado

\section{Discussão}

Os achados do presente estudo apontam que a prevalência de TMC foi de 40,0\% na amostra pesquisada, resultado semelhante a outros estudos brasileiros $(13,14)$ apresentando maior prevalência no gênero feminino, dados coerentes com a literatura (4). De fato, a maioria dos estudantes que buscam acompanhamento em saúde mental são do gênero feminino (15). Tal prevalência pode ter suas causalidades advindas de diferentes fatores, porém acredita-se que as universidades possuem dificuldades para cobrir a demanda referente à adaptação dos estudantes ingressantes à nova fase universitária (16) podendo levar a condições agravantes do estado emocional.

A insatisfação com o curso pode levar ao aumento dos distúrbios psicossomáticos, estresse e falta de confiança, o que tem sido associado à diminuição da capacidade e desempenho acadêmico (15). No presente estudo, os alunos com menor satisfação com a escolha profissional apresentaram maior prevalência de TMC. A esse respeito, mais da 
metade da amostra já pensou ou pensa em abandonar o curso, podendo ser consequência da insatisfação com o mesmo. Em particular, problemas de relacionamento com colegas, dificuldade de fazer amigos e pouca percepção de amizade aumentam a incidência de TMC e a probabilidade de abandono do curso (17). Em concordância a isso, os alunos com queixas de dificuldade de fazer amigos apresentaram maior prevalência de TMC, o que não surpreende, visto a importância das relações sociais para a saúde mental (18). Em geral, a transição e adaptação à universidade extrapolam tarefas curriculares e envolvem o manejo de habilidades sociais por parte do estudante, o que implica na necessidade de interações sociais e relações de interdependência (16). Não obstante, alunos que apresentam humor deprimido manifestam déficits, de maneira generalizada, para interações sócias (19).

Não surpreende, assim, que a criação de espaços de atendimento em saúde mental é uma proposta de intervenção que parece oportuna e extremamente necessária (16). No presente estudo, dos participantes com escores para TMC, 63,6\% e 83,3\% deles relataram não receber apoio emocional e sentem-se rejeitados por amigos e colegas, respectivamente. A associação dessa variável com a presença de TMC converge com achados de um outro importante estudo que demonstrou que poucas interações sociais e a consequente baixa percepção de apoio emocional está fortemente relacionada com o desenvolvimento de problemas físicos e mentais (18). Complementarmente, dados sugerem que a identificação de redes de apoio pelo estudante ameniza as consequências da transição escola-universidade, resultando em menores níveis de ansiedade, depressão e somatizações (13). Aqui, relata-se que 88,8\% dos estudantes do Grupo TMC alegaram não receber nenhum atendimento em saúde mental, como atendimento psicológico ou psiquiátrico especializado. Nesse sentido, temos um importante tópico de discussão: o papel da universidade em promover e divulgar espaços de auxílio aos acadêmicos, uma vez que, nas variáveis analisadas, os sujeitos que recebem apoio emocional, sentem-se acolhidos pelos amigos e colegas, não pensam em abandonar o curso, não apresentam dificuldade em interações sociais e sentem-se satisfeitos com a escolha profissional,apresentam menores prevalências de TMC. Curiosamente, alunos que experimentam sintomas de ansiedade e depressão, sintomas classicamente presentes nos TMC, tendem a procurar com maior frequência atendimento em saúde mental (15), sendo que, no presente estudo, não observou-se tal ação. Thomas e colaboradores sugerem que os estudantes que buscaram atendimento em saúde mental foram aqueles que reconheceram seus sintomas, bem como os benefícios da ajuda profissional (20). A literatura aponta que algumas terapias apresentam mais efetividade para a diminuição dos sintomas depressivos, ansiosos e somatoformes, característicos dos TMC (21). Salienta-se a Terapia Cognitivo-Comportamental, a qual vem demonstrando evidências consistentes quando utilizada como estratégia de atendimento em saúde mental (22). Os resultados do estudo podem contribuir com a promoção de políticas que visam a disseminação de ambientes de escuta por parte da universidade, desenvolvendo e ampliando programas de prevenção e tratamento dos aspectos psicossociais dos acadêmicos.

\section{Considerações finais}

O presente estudo verificou a prevalência e os aspectos associados ao TMC em estudantes universitários em uma universidade localizada em Santo Ângelo, Rio Grande do Sul. Os dados indicam alta prevalência de TMC nos universitários e associação com algumas variáveis específicas. Acredita-se que, sabendo disso, torna-se viável a criação de estratégias para melhora e prevenção. Enfatiza-se o alto número de pessoas que apresentaram escores para TMC que não recebem atendimento especializado em Saúde Mental. Considerando achados prévios de outros estudos que demonstraram que a desinformação e a baixa percepção de sofrimento mental impossibilitam a busca de 
ajuda, torna-se explícito a necessidade do papel ativo das instituições de ensino superior em não só oferecer, mas divulgar espaços de escuta para os estudantes.

Enfatiza-se que o estudo não visou encontrar causas para os TMC, pois o caráter transversal e descritivo permite apenas associações. No entanto, o índice de significância estatística e a convergência a outros achados sugere relevância dos resultados. Ainda, procurou-se diminuir os vieses por meio de respostas objetivas, anonimato, preenchimento individual e em silêncio. Outras limitações podem ter afetado os resultados: como a complexidade dos sintomas como causa de possíveis respostas equivocadas, onde o sujeito pode sofrer de estresse leve, mas relacionar aos sintomas de TMC, bem como possíveis distrações durante a aplicação dos questionários em sala de aula, pois esse ambiente proporciona pouco controle de fatores que influenciam a concentração dos respondentes.

Os trabalhos iniciais sobre o TMC possuíram maior ênfase no aspecto epidemiológico de prevalência, dessa forma, acredita-se que o presente trabalho contribui significativamente por enfatizar aspectos psicossociais, incluindo a investigação sobre o acompanhamento em saúde mental nos universitários. Para futuros estudos, sugere-se a realização de pesquisas longitudinais para compreender o mesmo fenômeno, podendo evidenciar aspectos causais e a compreensão da pouca adesão ao acompanhamento em saúde mental.

Em suma, o presente trabalho demonstra a presença do sofrimento psíquico em contexto universitário, sendo que este pode se manifestar de maneira significativa, possivelmente gradativa, tendo aspectos psicossociais associados. Nesse sentido, a busca por atendimento em saúde mental pelos universitários, embora pequena, parece essencial. Acredita-se que a divulgação de dados epidemiológicos acerca das variáveis associadas, como o presente estudo o fez, possibilita um avanço nos estudos e trabalhos na grande área.

\section{Referências}

1. Vigo D, Thornicroft G, Atun R. Estimating the true global burden of mental illness. The Lancet Psychiatry 2016;3(2):171-8.

2. Vos T, Allen C, Arora M, Barber RM, Brown A, Carter A, et al. Global, regional, and national incidence, prevalence, and years lived with disability for 310 diseases and injuries, 1990-2015: a systematic analysis for the Global Burden of Disease Study 2015. Lancet 2016;388(10053):1545-602.

3. World Health Organization. Depression and other common mental disorders: global health estimates. World Heal Organ. 2017;1-24.

4. Steel Z, Marnane C, Iranpour C, Chey T, Jackson JW, Patel V, et al. The global prevalence of common mental disorders: A systematic review and meta-analysis 1980-2013. Int J Epidemiol 2014;43(2):476-93.

5. Prince M, Patel V, Saxena S, Maj M, Maselko J, Phillips MR, et al. No health without mental health. Lancet 2007;370(9590):859-77.

6. Hyman SE. Psychiatric drug development: diagnosing a crisis. Cerebrum 2013;2013:5.

7. Walkup JT, Albano AM, Ph D, Piacentini J, Birmaher B, Compton SN, et al. NIH Public Access. English J 2009;359(26):2753-66.

8. Kaczkurkin AN, Foa EB. Cognitive-behavioral therapy for anxiety disorders: an update on the empirical evidence. - Servier Res Gr Dialogues Clin Neurosci. 2015;17:337-46.

9. Vittengl JR, Jarrett RB. Major Depressive Disorder. Wiley Handb Cogn Behav Ther. 2013;1131-60.

10. Harding TW, Climent CE, De Arango M V., Baltazar J, Ibrahim HHA, Ladrido-Ignacio L, et al. Mental disorders in primary health care: A study of their frequency and diagnosis in four developing countries. Psychol Med 1980;10(2):231-41.

11. Mari JDJ, Williams P. A Validity Study of a Psychiatric Screening Questionnaire. Br J Psychiatry 1986;148:23-7. 


\section{J. P. Perini et al./Vittalle v. 31, n. 1 (2019) 44-51}

12. Goncalves DM, Stein AT, Kapczinski F. Performance of the Self-Reporting Questionnaire as a psychiatric screening questionnaire: a comparative study with Structured Clinical Interview for DSM-IV-TR. Cad Saude Publica 2008;24(2):380-90.

13. Costa AG da, Ludermir AB. Transtornos mentais comuns e apoio social: estudo em comunidade rural da Zona da Mata de Pernambuco, Brasil. Cad Saúde Pública 2005;21(1):73-9.

14. Facundes VLD, Ludermir AB. Transtornos mentais comuns em estudantes da área de saúde. Rev Bras Psiquiatr 2005;27(3):194-200.

15. Cerchiari EAN, Caetano D, Faccenda O. Utilização do serviço de saúde mental em uma universidade pública. Psicol Ciência e Profissão [Internet] 2005;25(2):252-65.

16. Osse CMC, Costa II da. Saúde mental e qualidade de vida na moradia estudantil da Universidade de Brasília. Estud Psicol 2011;28(1):115-22.

17. Lima MCP, de Souza Domingues M, de Abreu Ramos Cerqueira AT. Preval??ncia e fatores de risco para transtornos mentais comuns entre estudantes de medicina. Rev Saude Publica 2006;40(6):1035-41.

18. House JS, Landis KR, Umberson D, House JS, Landis IARLR, Umberson D. Social Relationships and Health. Science 1988;241(4865):540-5.

19. Bolsoni-Silva AT, Loureiro SR. O Impacto das Habilidades Sociais para a Depressão em Estudantes Universitários. Psicol Teor e Pesqui 2016;32(4):1-8.

20.Thomas SJ, Caputi P, Wilson CJ. Specific Attitudes Which Predict Psychology Students' Intentions to Seek Help for Psychological Distress. J Clin Psychol 2014;70(3):273-82.

21. Otte C. Cognitive behavioral therapy in anxiety disorders: Current state of the evidence. Dialogues Clin Neurosci 2011;13(4):413-21.

22.Hofmann S, Asnaani A, Vonk I, Sawyer A, Fang A. The efficacy of CBT: a review of meta-analyses. Cogn Ther Res 2012;36(5):427-40. 